\title{
Combination Therapy of Momordica charantia Linn. (Bitter Melon) with Antihyperglycemic Agent Gives Increased Antioxidative and Hepatoprotective Actions in Alloxan-induced Diabetic Rats
}

\author{
Monirul Islam ${ }^{1}$, Md. Saiful Islam², Shaheda Zannah² and Mamunur Rashid ${ }^{2}$ \\ ${ }^{1}$ Department of Pharmacy, Pabna University of Science and Technology, Pabna, Bangladesh \\ ${ }^{2}$ Department of Pharmacy, University of Rajshahi, Rajshahi-6205, Bangladesh
}

(Received: August 9, 2018; Accepted: September 13, 2018; Published: January 17, 2019)

\begin{abstract}
Bitter melon, a popular vegetable of the Asian and African community, is used as an antidiabetic herb for the management of diabetes and its associated complications. The fastest growing metabolic disorder, diabetes mellitus (DM), is now a major cause of morbidity and mortality in developed countries. All the associated complications produced in diabetes are mainly caused by the imbalance between generation of free radicals and the antioxidant systems like catalase (CAT) and super oxide dismutase (SOD) enzymes. Again increased level of serum glutamate pyruvate transaminase (SGPT) and serum glutamate oxaloacetate transaminase (SGOT) are considered a consequence of hepatocyte damage due to non-alcoholic fatty liver disease (NAFLD) and patients with diabetes have an increased risk NAFLD and steatohepatitis. The present study was aimed at evaluating the effects of fixed dose combination therapy of Momordica charantia crude methanolic extract with metformin on oxidative stress and liver dysfunction indices in alloxan-induced diabetic Swiss Albino rats. Various groups of alloxan-induced diabetic rats (AIDRs) were daily treated for four weeks with bitter melon extract (BME) [(300 mg/kg body weight (BW)], metformin [(15 mg/kg body weight $(\mathrm{BW})]$ and their combination dose $(150 \mathrm{mg} / \mathrm{kg} \mathrm{BW}+7.5 \mathrm{mg} / \mathrm{kg} \mathrm{BW})$, separately through oral gavage. In our study, we observed that combination therapy lowered SGPT \& SGOT level by $34.58 \%$ and $47.89 \%$, respectively and increased SOD \& CAT enzyme activity by $74.94 \%$ and $43.36 \%$, respectively in comparison with their diabetic control groups. The changes obtained from the combination therapy were significantly $(\mathrm{p}<0.05)$ better than those of metformin and BME therapy alone. These findings suggest that this fixed dose combination therapy is more potent than their individual monotherapy and diabetic patients with increased oxidative stress and liver complications may be benefited by using this treatment.
\end{abstract}

Key words: Diabetes, bitter melon, metformin, combination therapy, antioxidants and hepatoprotective activity.

\section{Introduction}

Diabetes is a heterogeneous group of diseases characterized by a state of chronic hyperglycemia which results in a number of secondary complications e.g. cardiovascular, neurological, hepatic, ocular and renal abnormalities (Thornalley et al., 1996). The worldwide figure of patients affected by diabetes is worsening day by day and in developing countries at least 1 in 10 deaths (aged 35-64 years) is attributable to diabetes (Wild et al., 2004; Singh et al., 2011).
Large evidence from different researches showed that disease complications were related to the oxidative stress which was induced by the generation of free radicals (Garg et al., 1996). The body's endogenous antioxidant enzymes e.g. super oxide dismutase (SOD), catalase (CAT), glutathione (GSH) etc. are responsible for the detoxidication of free radicals (Jacob, 1995). Increased productions of free radicals and reactive oxygen species (ROS) consume antioxidant defense mechanisms of the body, cause

Correspondence to: Mamunur Rashid; E-mail: mamun69jp@yahoo.com 
oxidative damage to the cell membrane and increase lipid peroxidation and these phenomenon ultimately lead to the pathogenesis of many diseases of known and unknown etiology (Sathishsekar and Subramanian, 2005). Chronic liver disease e.g. nonalcoholic fatty liver disease (NAFLD) and steatohepatitis are very common in patients having diabetes (Scheen, 2014). The blood SGOT and SGPT levels are elevated due to hepatocellular damage and these two enzymes are commonly measured clinically as indirect markers of liver inflammation or injury like NAFLD. Insulin resistance, increased proinflammatory cytokine production, oxidative stress and mitochondrial dysfunction all have been posed important pathophysiological mechanisms leading to hepatocyte damage (Day, 2002).

Presently control of DM relies on many chemicals and plant extracts and more than 400 traditional plant treatments for DM have been recorded (Singh et al., 2011). Many plant extracts and plant products have been shown to reduce oxidative stress significantly, which may be an important property of plant medicines associated with the treatment of several ill-fated diseases including diabetes (Ayodhya et al., 2010). Among these, M. charantia is a good example of folk medicine, which is consumed by diabetic patients in India with the belief that it has hypoglycemic, hypolipidemic and potential fighting power against oxidative stress and that's why it was selected for this study (Basch et al., 2003). A single antidiabetic medicinal herb may contain a complex mixture of phytochemicals and bioactivitie compounds, which have multiple benefits by targeting several metabolic pathways. For this reason, it can be used either alone or in combination with other herbs or an orthodox antidiabetic agent that can be thought of as a type of combination therapy. This type of combination therapy exhibits a better (synergistic) effect than either medicine alone. In the present study, we investigated the antioxidant and hepatoprotective effects of methanolic extract of M. charantia (bitter melon) by combining a fixed dose of this herb with a standard oral hypoglycemic agent metformin in AIDRs. The prime focus of our research work was to design and develop a cost effective, more efficacious and less toxic combination therapy which will provide beneficial effects in the management of DM and associated complications.

\section{Materials and Methods}

Selection of animal: Total 30 Swiss Albino male and female rats weighing about 110-140 gm (60-70 days old) were acclimatized to the new environmental condition for a period of 1 week. During the experimental period the rats were kept in a well-ventilated animal house at $25^{\circ} \mathrm{C}$ temperature and were supplied with standard pellets from icddr, $b$ and fresh drinking water. All the rats were kept in cages and maintained with natural $12 \mathrm{hrs}$ light and dark cycle. The experiments were performed based on animal ethics guidelines of institutional animal ethics committee principle.

Experimental induction of diabetes: All the rats of different groups except normal control were allowed to fast for $12 \mathrm{hrs}$ and rendered diabetic by intraperitoneal (IP) injection of a freshly prepared alloxan $(110 \mathrm{mg} / \mathrm{kg} \mathrm{BW})$ solution in distilled water (DW) after base line glucose estimation. The alloxan treated animals had access to food overnight and drink (10\% glucose) solution to overcome alloxaninduced hypoglycemia. After $48 \mathrm{hrs,} \mathrm{blood} \mathrm{glucose}$ level (BGL) was measured by using glucose test meter (Bioland G-423S Test Strip, Germany) of the blood sample collected from the tail vein of the rats. When the condition of diabetes was established animals with BGL above $11.1 \mathrm{mmol} / \mathrm{l}$ was selected for the study.

Preparation of BME and metformin: After purchasing of fresh green fruits of bitter melon from the local market, the authenticity of $M$. charantia was identified at the Department of Botany, Rajshahi University. The fruits were thoroughly washed, cut into thin slices, sundried and ground to coarse powder and used for cold extraction. After extraction with methanol, the yield was found about $10 \mathrm{ml}$ crude extract per 100 gm bitter melon powder. Concentrated BME was a viscous mass and freely soluble in water. BME dosage was produced in 
solution by using DW and administered at a dose of $300 \mathrm{mg} / \mathrm{kg}$ BW. As a standard oral hypoglycemic agent, metformin was used in this study. Before administration metformin was dissolved in DW and then administered at a dose of $15 \mathrm{mg} / \mathrm{kg}$ BW. The combination dose was prepared in solution form at a concentration of BME $(150 \mathrm{mg} / \mathrm{kg} \mathrm{BW})+$ metformin $(7.5 \mathrm{mg} / \mathrm{kg} \mathrm{BW})$. All the doses were given to the various animal groups once in every $24 \mathrm{hrs}$ for four weeks by using oral gavage.

Treatment of animals: In the current study protocol, rats were randomly assigned into 5 groups (A, B, C, D \& E) containing six rats in each group. The repeated dose treatment of animals began on the next day after diabetes induction (this was considered as 1st day) and continued for 28 days. The treated animal groups were as follows - Group A: Normal control; Group B: Diabetic control; Group C: Diabetic + Metformin (15 mg/kg BW); Group D: Diabetic + BME (300 mg/kg BW); Group E: Diabetic + Metformin $(7.5 \mathrm{mg} / \mathrm{kg} \mathrm{BW})+$ BME $(150 \mathrm{mg} / \mathrm{kg}$ BW).

After 28 days of treatment period, rats were again fasted for at least $16 \mathrm{hrs}$ and tested for their individual baseline BGL. The animals were then sacrificed after anesthesia by using chloroform and blood samples were collected directly from thoracic artery with heparinized syringe and centrifuged at $4000 \mathrm{rpm}$ for $30 \mathrm{~min}$. Serum was separated and then quickly stored at refrigerator for the analysis of antioxidant enzymes (SOD \& CAT) activity and liver dysfunction markers (SGOT \& SGPT) levels.

Biochemical analysis: The blood serum was examined for the estimation of liver functions abonormality markers like SGOT and SGPT levels by taking UV absorbance, using diagnostic kits (Human, Germany). Liver was isolated from the sacrificed animals and homogenized in chilled Tris buffer $(10 \% \mathrm{w} / \mathrm{v})$. The homogenate was then centrifuged at $4000 \mathrm{rpm}$ for $15 \mathrm{~min}$ in cold centrifuge and the supernatant was analyzed for SOD and CAT activity. The SOD activity was determined by the method of Kakkar et al. (1984) and CAT activity was assayed by the method of Sinha (1972).
Statistical analysis: The results are expressed as mean \pm SEM using Graph Pad Prism (version 4.0) computer program (Graph pad Software San Diego, CA, USA). We used a one-way analysis of variance (ANOVA), followed by Dunnett's post-hoc test or students paired or unpaired t-test where appropriate. The statistical method applied in each analysis was described in each figure. Results were considered to be significant when $\mathrm{p}$ values were less than 0.05 $(\mathrm{p}<0.05)$.

\section{Results and Discussion}

The effects of monotherpay of BME and metformin and their combination therapy on liver dysfunction indices like SGOT \& SGPT levels and antioxidant enzymes e.g. SOD \& CAT activities were investigated in long term AIDRs.

\section{Effects on liver dysfunction indices}

Effects on SGOT level: At the end of our four weeks treatment protocol with drugs when we tested SGOT level by using serum we found marked rise of SGOT (103.20\%) level in diabetic control group as compared to the normal group. Long term daily dose therapy of BME and metformin alone and their combination dose in other tested animal groups significantly $(\mathrm{p}<0.05)$ decreased SGOT level as compared to AIDRs as depicted in table 1.

Effects on SGPT level: We also observed raised SGPT value (125.14\%) as like as SGOT level in the AIDRs in comparison to the normal control group. After the long term repeated dose treatment with metformin, BME and their combination in other diabetic animal groups showed significant $(p<0.05)$ reduction of SGPT level. However, in this case therapy with metformin alone responded more effectively than the combination therapy. All these results are outlined in table 1.

\section{Effects on antioxidant enzyme activities}

Effects on SOD activity: The variation of values of SOD enzyme activity at the end of four weeks repeated dose treatment with metformin, BME and combination of both normal and AIDRs are depicted 
in table 2. Animals of diabetic control group had shown decreased level SOD enzyme activity $(48.65 \%)$ in comparison with normal control group. We found at the end of the study that, metformin, BME and their combination exclusively increased
SOD enzyme activity by $34.17,24.60$ and $74.94 \%$, respectively. Form the data, it was cleared that combination therapy expressed most prominent efficacy than that of monotherapy in case of increasing SOD enzyme activity in AIDRs.

Table 1. SGOT and SGPT levels in normal and AIDRs after four weeks treatment.

\begin{tabular}{lcccc}
\hline \multirow{2}{*}{ Groups } & \multicolumn{2}{c}{ SGOT (units/l) } & \multicolumn{2}{c}{ SGPT (units/l) } \\
\cline { 2 - 4 } & Mean \pm SEM & $(\%)$ Inhibition & Mean \pm SEM & (\%) Inhibition \\
\hline Normal & $32.55 \pm 1.69$ & & $21.12 \pm 0.80$ & \\
AIDRs & $66.14 \pm 1.09^{\#}$ & & $47.55 \pm 1.02^{\#}$ & 41.01 \\
Diabetic + Metformin & $48.86 \pm 1.33^{*}$ & 26.12 & $28.05 \pm 0.52^{* *}$ & 21.20 \\
Diabetic + BME & $44.06 \pm 0.86^{*}$ & 33.38 & $37.47 \pm 0.78$ & 34.58 \\
Diabetic + Metformin + BME & $34.47 \pm 1.62^{* *}$ & 47.89 & $31.11 \pm 1.23^{*}$ & \\
\hline
\end{tabular}

The data are presented as mean \pm SEM; $\mathrm{n}=5$ in each group, ${ }^{*} \mathrm{p}<0.05, * * \mathrm{p}<0.01$ compared to diabetic control group (One way ANOVA followed by Dunnett's test) and \#p $<0.05$ vs. normal group.

Table 2. Data for the SOD and CAT enzyme activities in normal and AIDRs after four weeks treatment.

\begin{tabular}{|c|c|c|c|c|}
\hline \multirow[t]{2}{*}{ Groups } & \multicolumn{2}{|c|}{$\begin{array}{l}\text { Super oxide dismutase } \\
\text { (U/mg protein) }\end{array}$} & \multicolumn{2}{|c|}{$\begin{array}{c}\text { Catalase (CAT) } \\
(\mu \mathrm{mol} / \mathrm{min} / \mathrm{mg} \text { protein })\end{array}$} \\
\hline & Mean \pm SEM & (\%) Increased & Mean \pm SEM & (\%) Increased \\
\hline Normal & $8.55 \pm 0.18$ & & $83.78 \pm 1.01$ & \\
\hline AIDRs & $4.39 \pm 0.14^{\#}$ & & $52.21 \pm 1.33^{\#}$ & \\
\hline Diabetic + Metformin & $5.89 \pm 0.38^{*}$ & 34.17 & $61.12 \pm 0.91^{*}$ & 17.07 \\
\hline Diabetic + BME & $5.47 \pm 0.09^{*}$ & 24.60 & $63.93 \pm 1.74^{*}$ & 22.45 \\
\hline Diabetic + Metformin + BME & $7.68 \pm 0.13^{* *}$ & 74.94 & $74.85 \pm 1.85^{* *}$ & 43.36 \\
\hline
\end{tabular}

The data are presented as mean \pm SEM $; n=5$ in each group, $* p<0.05, * * p<0.01$ compared to diabetic control group (One way ANOVA followed by Dunnett's test) and \#p $<0.05$ vs. normal group.

Effects on catalase enzyme activity: As of SOD enzyme activity, AIDRs also showed significant decline in CAT activity (37.68\%) in comparison with their normal control groups after long term study. At the completion of the experiment, the percentage of increment of CAT enzyme activity by the treatment of metformin, BME and combination in different animal groups as compared to diabetic control group are shown in table 2. Here, we also found potent activity of combination therapy than that of metformin and BME therapy alone. Metformin, BME and their combination increased CAT activity in AIDRs by $17.07,22.45$ and $43.36 \%$, respectively.
Diabetes mellitus comprises a heterogeneous group of disorders characterized by high BGL which results in dyslipidemia and oxidative stress (Chaturvedi and George, 2010) and leads to different types of complications such as atherogenesis, hepatocellular damage, cardiovascular disorders, neuropathy and retinopathy, mainly due to an imbalance between generations of free radicals and the antioxidant system (Peerapatdit et al., 2006). The present long term studies on the combination therapy of BME with metformin provided some light on the beneficial effects like antioxidant and hepatoprotective actions in normal and AIDRs. IP injection of freshly prepared alloxan monohydrate $(110 \mathrm{mg} / \mathrm{kg}$ 
BW) solution was used to induce diabetes (BGL $\geq$ $11.1 \mathrm{mmol} / \mathrm{l}$ ) among the 16 hours fasted rats (Zannah et al., 2015). Alloxan selectively kills the pancreatic $\beta$-cells because it preferentially accumulates in $\beta$ cells through uptake via the GLUT2 glucose transporter. The resulting insulinopenia causes a state of experimental DM called 'alloxan diabetes' (Dunn et al., 1943) which results a decrease in endogenous insulin release. Hypercholesterolemia and hypertriglyceridemia are common complications of DM in addition to hyperglycemia. In our previously published research report we discussed the significant antihyperglycemic and hypolipidemic effects of BME and metformin combination therapy in normal and AIDRs (Islam et al., 2018).

The enzymes SGPT is a cytosolic enzyme primarily present in the liver and SGOT is a mitochondrial enzyme released from heart, liver, skeletal muscle and kidney. Both of these enzymes are the most sensitive markers employed in the diagnosis of liver diseases. When liver cells are injured or died, these enzymes can leak through the liver cell membrane into the blood circulation and serum levels become increase (Zannah et al., 2015). As diabetic rats show a significant increase in these liver markers so it is conclusive that diabetes is associated with hepatocellular damage (Thenmohzi and Subramanian, 2009). Long term oral treatment of combination therapy decreased the serum SGPT and SGOT levels in alloxan induced diabetic rats when compared to their diabetic group. Though combination therapy showed moderate decrease in SGPT level but showed significant $(\mathrm{p}<0.05)$ decrease in SGOT level than therapy with metformin and BME alone. These results suggest that combination therapy offers protection by preserving the structural integrity of the hepatocellular membrane and our findings are in agreement with the previously published results (Abd El Sattar El Batran et al., 2006).

Hyperglycemia results in oxidative stress which causes free radical formation through various biochemical reactions. Glucose can undergo autooxidation and generate hydroxyl free radicals
(OH) (Turko et al., 2001) which again causes autooxidation of unsaturated lipids in plasma and cell membrane. Furthermore, enhanced metabolism of glucose through polyol (sorbitol) pathway depletes glutathione reserves and enhanced production of free radicals (Amira, 2010). These consequences ultimately lead to pathogenesis of many diseases including cardiovascular disease (CVD). The endogenous antioxidant enzymes like SOD and CAT are responsible for the detoxification of deleterious oxygen radicals. The activity of SOD and CAT decreases due to inactivation by $\mathrm{H}_{2} \mathrm{O}_{2}$ or by glycation of enzymes which is observed in diabetes and this may result in a number of deleterious effects due to accumulation of superoxide radicals and $\mathrm{H}_{2} \mathrm{O}_{2}$. SOD is the most important enzymes in the enzymatic antioxidant defense system which catalyses the dismutation of superoxide radicals to produce $\mathrm{H}_{2} \mathrm{O}_{2}$ and molecular oxygen, hence diminishing the toxic effects caused by their radical. Again CAT catalyses the reduction of $\mathrm{H}_{2} \mathrm{O}_{2}$ and protects the tissues from highly reactive $\mathrm{OH}$ radicals (Lin et al., 2005). In our study, long term induction of diabetes with alloxan results in a significant reduction in SOD and CAT activity in comparison with normal rats. Administration of $M$. charantia extract effectively normalizes this impaired antioxidant status in diabetic rats. Treatment with BME and metformin increased the activity of these antioxidant enzymes in comparison to their diabetic control rats where BME alone showed more prominent effect in increment of CAT activity but less prominent effect in increment of SOD activity compared to metformin alone. However, combination therapy increased the SOD and CAT activity most significantly $(\mathrm{p}<0.05)$ than those of monotherapy (Sathishsekar and Subramanian, 2005). Different researches have demonstrated that BME increased activity of SOD and CAT by accelerating the catabolism of $\mathrm{H}_{2} \mathrm{O}_{2}$ in diabetic rats due to its superoxide scavenging and powerful antioxidant activities (Thenmohzi and Subramanian, 2009). It may also reduce the potential glycation of these enzymes or reduce reactive oxygen free radicals and improve the activities of other antioxidant enzymes. 


\section{Conclusion}

The results obtained from our current study indicate that application of BME and metformin in AIDRs showed therapeutically beneficial effects and their combination therapy increased those effects more strongly. As a plant crude extract, BME contained a mixture of complex phytochemicals like cucurbitane-type triterpene glycosides, flavonoids which has hypoglycemic, powerful antioxidative and hepatoprotective actions. These actions became more potent in safe and synergistic combination of BME with metformin and our findings from this study clearly indicated that this combination therapy has the ability to give hepatic protection and handle oxidative stress which can reduce the risk parameters associated with diabetes. From our investigations we can suggest that co-administration of oral hypoglycemic agent metformin with crude extract of $M$. charantia may be more beneficial in management of diabetes with other associated complications like liver disorders and increased oxidative stress than either drug alone. However, but further detailed long term and placebo controlled human volunteer trials are required to prove the clinical appropriateness of this fixed dose combination therapy.

\section{Acknowledgement}

The authors are thankful to Square Pharmaceuticals Ltd., Bangladesh for the kind gift of metformin.

\section{References}

Amira, A.M. 2010. Oxidative stress and disease. Res. J. Iimmunol. 3, 129-145.

Ayodhya, S., Kusum, S. and Anjali, S. 2010. Hypoglycemic activity of different extracts of various herbal plants. Inter. J. Res. Ayurv. Pharm. 1, 212-224.

Abd El Sattar El Batran, S., El-Gengaihi, S.E. and El Shabrawy, O.A. 2006. Some toxicological studies of Momordica charantia L. on albino rats in normal and alloxan diabetic rats. J. Ethnopharmol. 108, 236-242.

Basch, E., Gabardi, S. and Ulbricht, C. 2003. Bitter melon (Momordica charantia): A review of efficacy and safety. Am. J. Health Syst. Pharm. 60, 356-359.
Chaturvedi, P. and George, S. 2010. Momordica charantia maintains normal glucose levels and lipid profiles and prevents oxidative stress in diabetic rats subjected to chronic sucrose load. J. Med. Food. 13, 520-527.

Day, C.P. 2002. Pathogenesis of steatohepatitis. Best Pract. Res. Clin. Gastroneterol. 16, 663-678.

Dunn, J.S. and McLetchie N.G.B. 1943. Experimental alloxan diabetes in the rat. Lancet. 245, 384-387.

Islam, M., Islam, M.S., Zannah, S., Sadik, G. and Rashid, M. 2018. Momordica charantia (Bitter melon) in combination with metformin potentiates hypoglycemic and hypolipidemic effects in alloxan-induced diabetic rats. Bangladesh Pharm. J. 21, 109-117.

Jacob, R.A. The integrated antioxidant system. Nutr. Res. 15, 755 .

Garg M.C., Ojha, S. and Bansal, D.D. 1996. Antioxidant status of streptozotocin diabetic rats. Ind. J. Exp. Biol. 34, 264-266.

Kakkar, P., Das, B. and Viswanathan, P.N. 1984. A modified spectrophotometric assay of superoxide dismutase. Ind. J. Biochem. Biophys. 21, 130-132.

Lin, Y.F., Tsai, H.L., Lee, Y.C. and Chang, S.J. 2005. Maternal vitamin E supplementation affects the antioxidant capability and oxidative status of hatching chicks. J. Nutr. 135, 2457-2461.

Peerapatdit, T., Patchanans, N., Likidlilid, A., Poldee, S. and Sriratnassathavorn, C. 2006. Plasma lipid peroxidation and antioxidant nutrients in type 2 diabetes patients. J. Med. Assoc. Thai. 89, S147-S155.

Sathishsekar, D. and Subramanian, S. 2005. Antioxidant properties of Momordica charantia (bitter gourd) seeds on streptozotocin induced diabetic rats. Asia. Pac. J. Clin. Nutr. 14, 153-158.

Scheen, A.J. 2014. Pharmacokinetic and toxicological considerations for the treatment of diabetes in patients with liver disease. Expert Opin. Drug Metab. Toxicol. 10, 839-857.

Sinha, A.K. 1972. Colorimetric assay of catalase. Anal. Biochem. 47, 389-94.

Singh, J., Cumming, E., Manoharan, G., Kalasz, H. and Adeghate, E. 2011. Medicinal chemistry of the anti-diabetic effects of Momordica charantia: active constituents and modes of actions. Open Med. Chem. J. 2, 70-77.

Thenmohzi, A.J. and Subramanian, P. 2009. Antioxidant potential of Momordica charantia in ammonium chloride-induced hyperammonemic rats. Evid. Based Complement. Alternat. Med. 2011, 1-7. 
Thornalley, P.J., McLellan, A.C., Lo, T.W., Benn, J. and Sönksen, P.H. 1996. Negative association between erythrocyte reduced glutathione concentration and diabetic complications. Clin. Sci. 91, 575-582.

Turko, I.V., Marcondes, S. and Murad, F. 2001. Diabetes associated nitration of tyrosine and inactivation of succinyl-CoA: 3-oxoacid CoA-transferase. Am. J. Physiol. Heart Circ. Physiol. 281, H2289-H2294.
Wild, S., Roglic, G., Green, A., Sicree, R. and King, H. 2004. Global prevalence of diabetes: Estimates for the year 2000 and projections for 2030. Diabet. Care. 27, 1047-1053.

Zannah, S., Islam, M., Yusuf, A., Asaduzzaman, M., Shahid, S.M., Al Bari, A.A. and Rashid, M. 2015. Antioxidant in combination with hydroxychloroquine improve antioxidant and hepatoprotective activities in alloxan-induced diabetic rats. Bangladesh Pharm. J. 18, 72-77. 